\title{
The visual sociogram in qualitative and mixed-methods research
}

Paola Tubaro, CNRS and University of Greenwich, p.tubaro@gre.ac.uk (corresponding author)

Louise Ryan, University of Middlesex, I.ryan@mdx.ac.uk

Alessio D’Angelo, University of Middlesex, a.dangelo@mdx.ac.uk

\begin{abstract}
The paper investigates the place of visual tools in mixed-methods research on social networks, arguing that they can not only improve the communicability of results, but also support research at the data gathering and analysis stages. Three examples from the authors' own research experience illustrate how sociograms can be integrated in multiple ways with other analytical tools, both quantitative and qualitative, positioning visualization at the intersection of varied methods and channelling substantive ideas as well as network insight in a coherent way.

Visualization also facilitates the participation of a broad range of stakeholders, including among others, study participants and non-specialist researchers. It can support the capacity of mixedmethod approaches to research areas of the social that would otherwise be difficult to reach, such as hidden populations and informal organisations. On this basis, visualization appears as a unique opportunity for mixing methods in the study of social networks, emphasizing both structure and process at the same time.
\end{abstract}

\section{Keywords}

Social networks, mixed methods, qualitative research, visualization, sociograms, relational data. 


\section{Introduction}

Data visualization and infographics are enjoying ever greater popularity in fields as diverse as science, journalism, and the arts, but less so in social science, where they are used primarily for communication of final outcomes, and to a much lesser extent for (exploratory) data analysis (Healy \& Moody 2014). One exception is the study of social networks, where visualization of actors as points, and of ties between actors as lines joining up their endpoints, has always played a prominent role (Freeman 2000). Diagrams of social connections have been in use since the ground-breaking work of, among others, Jakob L. Moreno (1934) who credited them for the very first successes of social networks research and coined the word "sociogram". Since then, visualisation has accompanied the development of the field, and is evolving at unprecedented pace today (Brandes et al. 2006; Brandes et al. 2013).

Traditionally, network visualizations served for basic, descriptive purposes, whilst more advanced analytical results were often given in verbal or tabular form (Brandes et al. 2001: 2). Recent work aims to better integrate formal analysis and visual presentation, devising rigorous graphical solutions to show key relationships between the data and link them to indicators that support explanations (Correa \& Ma 2011). The production of network graphics shifts "from the artistic to the scientific domain, the implications being increased reliability and easy reproducibility" (Brandes et al. 2001: 3).

This evolution involves a move away from the hand-drawn diagrams of Moreno and other pioneers, guided by intuitive concepts, and a progressive shift to automatic software tools, based on insight from graph theory, a branch of mathematics, and algorithmics, a growing area within computer science. Visual representations are now possible even for very large, multi-dimensional and complex networks, and the word "sociogram" has been largely replaced by "graph". In conjunction with advances in measurement of network structural properties and in statistical inference for relational data, these developments have appreciably supported quantitative research.

We contend in this article that visualisation has also a momentous role to play in qualitative and mixed-methods social networks research. Rooted in early ethnographic studies of the structures of kinship and relationships carried out by Barnes (1954), Bott (1957) and Mitchell (1969), qualitative social networks research places less emphasis on structure and more on content of ties, such as their meanings, interpretations, and stories (Crossley 2010). Initially less prominent than quantitative social network analysis, qualitative research is progressively coming to the fore (Edwards 2010; Crossley 2009; Emmel \& Clark 2009; Heath et al. 2009). In fact, this tradition has been fertile ground for remarkable developments in the use of visual tools in data gathering and data analysis - areas that are otherwise underdeveloped in the social sciences.

To illustrate the power and potential of visualization in qualitative and mixed-methods social networks research, we rely on three examples from our own experience, each of which involves different usages of visuals, different solutions to integrate data collection with analysis and presentation of results, and different ways of positioning visualization at the intersection of varied methods.

The first example demonstrates how use of a network-drawing exercise within an in-depth interview can transform the encounter and elicit richer data. The joint analysis of visuals and narratives offers a systematized, holistic view of relationships, and provides more comprehensive and far-reaching insight than conventional qualitative methods. It can also capture aspects of social dynamism changes in relationships or the movement of connections across different social contexts (family, school, workplace, neighbourhood) over time, or encompassing multiple contexts as a result of an actual social process. Visualization integrates seamlessly with fieldwork research, in a mixed-methods approach that derives understanding from both structure and content of social ties, as portrayed and talked through by participants. 
The second example confirms that a network-drawing exercise benefits data collection, not only in qualitative but also in quantitative approaches: this time, it was embedded in a self-administered, computer-based questionnaire, aiming to facilitate respondents' experience and increase completion rates. Analysis can also benefit from visuals, but does not need to rely on participants' drawings highly idiosyncratic and necessarily incomplete without extra information on, for example, the nature of the relationships and the modes of interaction. To produce visuals that would support analysis, this project used an algorithm implemented in software to convert data (participants' drawings and their responses to the questionnaire) into standardized images enabling systematization, comparison and the drawing of typologies. Different visualizations reveal different aspects of social networks and assist at different stages of the research process.

The third example also uses algorithmic tools to produce images - but the underlying data in this case were qualitative rather than quantitative, in an interpretive effort to reconstitute the essential features of an informal network with ill-defined boundaries. Here, visual tools prove their capacity to organize and give coherence to heterogeneous information that would escape a purely quantitative study, assisting progressive refinements of the analysis in an iterative process whereby participants' feedback on the researcher's preliminary layouts prompted corrections, until a final result was achieved. Algorithms do not necessarily require highly structured data and can be adapted to serve the needs of qualitative and mixed-methods approaches; their use does not need to be limited to researchers' analysis in the lab, but can support further interaction with study participants.

All three cases show that network visualization bolsters dialogue with study participants. By nudging them towards thinking in systemic terms, visuals empower them while also enriching and deepening the information they provide to the researcher. In this sense, free drawings inspired by participants' imagination still have a role to play together with algorithms and graph-theoretic maps. Accordingly, we insist on using the older word "sociogram" in light of its openness to use of a diverse range of techniques - not only high-tech, computer-based visual software but also manual, even paper-andpencil drawings.

While the three cases have different substantive objects (migration, health, organisations), they share a common concern to develop visualization as a unique opportunity for mixing methods in the study of social networks, responding to recent calls to view networks as both structure and process (Edwards 2010), beyond the conventional quantitative-qualitative duality. Before presenting and discussing the three cases, the next two sections examine the key implications of using visualisation to engage with and empower participants and to create "interfaces" between different methods.

\section{Visualisation and sensory expression: bringing networks to study participants}

Social networks are not "out there" for all to see, but are rather to be understood as constructs, models and abstractions that researchers use to describe and understand aspects of empirical social reality (Peay 1980). Mapping social networks is a way to uncover how interdependencies, associations, relationships and interactions shape society, overcoming standard sociological representations of independent actors as sets of individual attributes (what Crossley \& Edwards, this issue, refer to as "variable analysis").

Similarly network data, like all social data, are not simply "given" by actual phenomena, but are selectively harvested according to pre-defined notions and theories (Kitchin 2014: 2). Specifically, the construction of a network requires thoughtful choice of actors (for example, whether persons or organisations), relationships (friendship, advice, co-work), and measures (simple presence/absence of a tie or further qualifications). The resulting actor-tie data represent interdependencies that underpin social structures. Highly abstract, this approach is also technically complex as the size of the dataset grows exponentially with the number of actors: with as few as ten actors, the existence (and possibly, qualifications) of ninety potential ties must be established, tracked, and understood. 
By appealing to the senses, visualization makes such constructs and mental representations accessible, helping viewers grasp them. Reflecting "the desire to take what is normally outside the scale of human senses and to make it visible and manageable" (Manovich 2002: 8) and structuring experience in perceptual ways, visualization allows "the comparison of a set of values, the illustration of relationships between data points, the indication of the parts of a system and the relationship and interaction of these parts, the creation and interpretations of maps, the tracking of change over time" (Diamond 2010: 1). This is crucial in the study of social networks: "if the research topic is fundamentally interpersonal (such as influences on migration choices or political opinion) then [...] network visualization provides a perspective on these topics that cannot be gained otherwise" (McCarthy et al. 2007: 159).

The capacity of visualization to appeal to the senses allows usage with laypersons, and undeniably throughout their history, sociograms have been primarily used as presentation tools - the end of the research pipeline - directed at the final beneficiaries of a study, be they funders, businesses, policy makers or the general public. Another type of laypersons - at the start of the pipeline - are study participants, and indeed some social network researchers have also used sociograms with them, thereby extending the use of visualisation to data collection, in addition to presentation of results.

One way of doing so uses algorithmic visualisation, whereby the researcher first asks participants to name and qualify their network members, then uses the data to visually represent the network in points-and-lines format (typically with some rule implemented in software) and subsequently shows the result to respondents, inviting their comments (McCarthy et al. 2007; Bellotti 2008). An alternative use of sociograms in data collection in real-time asks respondents to directly draw a network, freely or in some pre-defined framework: put differently, it uses the graphical form as the very tool through which data are elicited, instead of deriving it from a list of names and qualifications (Hogan et al. 2007; Ryan et al. 2014; Tubaro et al. 2014).

Either way, visualisation gives participants a more global view of their relationships and the social contexts they are embedded in, a view that would not emerge spontaneously from just describing their relationships in interviews, or adding names of contacts to a list in a questionnaire. Complex social structures are made intelligible in their sensory expression through the sociogram, and unanticipated aspects come to the fore, stimulating reflection (McCarthy et al. 2007: 153-154). With sociograms, participants move "from description of social practices, to their elaboration and theorisation" (Emmel \& Clark 2009: 16).

This can be seen as a form of empowerment of participants, enacting a form of "data-subjectivity" (Manovich 2002): the personal, daily experience of immersion in flows of data (Liu 2004) and navigation in data so common in the digital world of today. Data, it is often said, are no longer a purely abstract entity, and with the rising popularity of social media, even network data and their graphical representations have become familiar to many.

More importantly, the combination of visual and interview/questionnaire data gathering techniques is in and by itself, a mixed-methods approach. It supports deeper understanding of networks, allowing at the same time to systematise information on ties and to investigate their contents and meaning (Bellotti 2014).

\section{Visualisation and analysis: the sociogram at the interface between methods}

The sociogram coalesces different data sources at collection stage, and stands at the interface between different methods at analysis stage. Technically, network visualizations are produced as transformations whereby, after representing all data using a standard code, one representation - a dataset - is mapped into another - an image (Manovich 2002). Constant dialogue with the data from which the sociogram is produced (or with the data it enables to elicit) is essential to give it meaning. By combining the two, the researcher derives insight to explain how and why the displayed graphs 
look as they do, and what underlying social practices, dependencies and interactions they actually reveal. It is in this sense that, as Moreno first argued, "the sociogram [...] is more than merely a method of presentation. It is first of all a method of exploration." (1953: 95-96).

Taken out of context, without this broader framework, visualization might not always be helpful. The relative arbitrariness of coding and the forms of reduction inherent in mapping and imaging, where the relations in data are typically represented with a small number of geometric primitives (Manovich 2010), may constrain imagination and even generate incorrect impressions. Constraints emerge when the drawing of the network is experienced as a restriction that imposes pre-defined patterns and orients reflection too prescriptively. The potential to mislead viewers is a concern because, as discussed earlier, graphs appeal to senses, and sensory experience suggests that visual clues may be meaningful, although this is not always true; for example, the points at the centre of the graph may not be the most central in terms of the structure of their connections to others.

To produce meaningful sociograms, Brandes et al. (2013) insist on substantively-informed designs. Substance involves enriching the graphs with the questioning that originally motivated the research interest of those who collected the data, and with any additional insight generated as a result of the analysis. In quantitative research, this means joint use of sociograms and statistical graphs such as histograms or scatterplots, or development of sophisticated designs that filter and position points (network members, i.e. social actors) based on some computed structural properties. With geolocalised data, this may involve mapping networks onto physical spaces, to reveal how geographical and relational distances impinge on each other. The recent development of these techniques has made social network analytics and visualization more interwoven, with visualization no longer seen as a passive process that produces images from an existing set of (numerical) results, but feeding directly into the researchers' understanding of network structure, composition, and dynamics (Correa \& Ma 2011).

Substance-based designs are also relevant for mixed-methods and qualitative research. Beyond standard points-and-lines language, different elements may be added: for example, rings that assemble actors together according to shared affiliation to a social context (for example school, workplace), or some relevant affinity. By distinguishing relationships from the contexts in which they arise, the researcher can disentangle contextual from relational effects, and can detect the trajectories through which relations evolve, become autonomous from the context (e.g. schoolmates who remain friends after leaving school), or overlap across different contexts (Bidart et al. 2011).

An example of a substance-based design is the "target" sociogram pioneered by Mary Northway as far back as 1940. It ranks network members based on a substantively relevant notion of prominence and uses the geometric metaphor of concentric circles to map this ranking to a graphical space, so that more prominent actors are positioned in the inner circles and those less prominent in the outer circles. It can be extended to use rigorous centrality metrics (Brandes et al. 2003), but its success relies on the self-explanatory association between spatial and relational concepts, allowing use even with children (Samuelsson et al. 1996). Its simplicity makes it ideal for collecting data "in an intuitive and easy way" (Carrasco et al. 2006: 9). Target sociograms have been utilized in qualitative and mixed-methods studies on issues as diverse as social support (Antonucci 1986, Pahl \& Spencer 2004), communication media (Hogan et al. 2007), migration (Ryan et al. 2014) and online communities (Tubaro et al. 2014).

With substance-based designs, the sociogram intervenes at data analysis stage, while at the same time allowing the results of the analysis to feed back onto sociogram design and potentially, data collection. The sociogram, then, appears to sit at the intersection between different data and methods, relates different stages of the research, and facilitates contact between different stakeholders. The best way to expound this view is by way of example, and we now do so by discussing our three extended case studies. 


\section{The dynamic interplay between visual and narrative data}

Our first example draws on Louise Ryan's experience of adapting Northway's "target" sociogram to studies of personal networks, aiming to uncover the set of family members, friends, and acquaintances surrounding a focal individual. Here, the suite of concentric circles represents the personal network, where the person of interest ("ego") is at the centre, and places their contacts ("alter") around them according to their prominence, interpreted as perceived relational proximity, so that closer alters will be at the interior and less close ones on the outside. The social contexts and nature of the relationships (family, friendship, business etc.) can be represented as sectors in the diagram. While loosely reminiscent of Norbert Elias" "naively egocentric" (1970: 14) view of social life, which also uses a concentric-circles design, this representation of the personal network should not be seen as a form of reification that assumes the existence of social structures as pre-defined objects external to, and exerting pressure on, the individual. On the contrary, it aims precisely to draw on the experience and the perception of the individual to reconstitute the social relations and human interdependencies that shape social structures, under a minimum assumptions and constraints.

The target sociogram can be used in fieldwork, asking the respondent to complete either a paperand-pencil or a computer-based version of it. It has been suggested that paper diagrams are less threatening, more user-friendly (allowing for easy visualization), and not vulnerable to technical failures (Cheong et al. 2013). Having used paper-based ego-net sociograms in two research projects (for a discussion see Ryan et al. 2014), in this section, Ryan argues that incorporating this visual tool into a face-to-face interview encounter not only provides a valuable insight into the process of network visualization but also offers rich data on network composition as well as on the meaning and dynamics of relationships.

In 2014, emerging from previous work on Polish migrant networks (Author 2 et al. 2008; Author 2 2011), Ryan interviewed 20 Polish men and women who had lived in London for approximately ten years (since Polish accession to the EU in 2004). Using sociograms embedded in biographical, face-toface interviews, the study aimed to understand how migrants created, sustained and changed social relationships over time. This particular version of the sociogram consisted of three concentric circles divided into four quadrants (friends, family, work, neighbours/ hobbies/ other) and was adapted from Hersberger (2003, see also Tapini forthcoming). An example of such a sociogram is presented in Figure 1.

Interviews lasted approximately one hour and began by asking about migration processes; when, how and why people migrated. Inter-personal relationships were usually central to this story. Changing relations with friends, partners, relatives were woven through the narrative. Thus, networks were discussed in some detail before the sociogram was introduced - about 15 minutes into the interview. The sociogram took participants approximately 15 to 20 minutes to complete interspersed with discussion (and several probes and prompts from the interviewer). Upon completion the sociogram was left on the table while the interview moved on to other topics, such as future migration plans. It is noteworthy that many participants went back to the sociogram throughout the rest of the interview to add extra alters. 


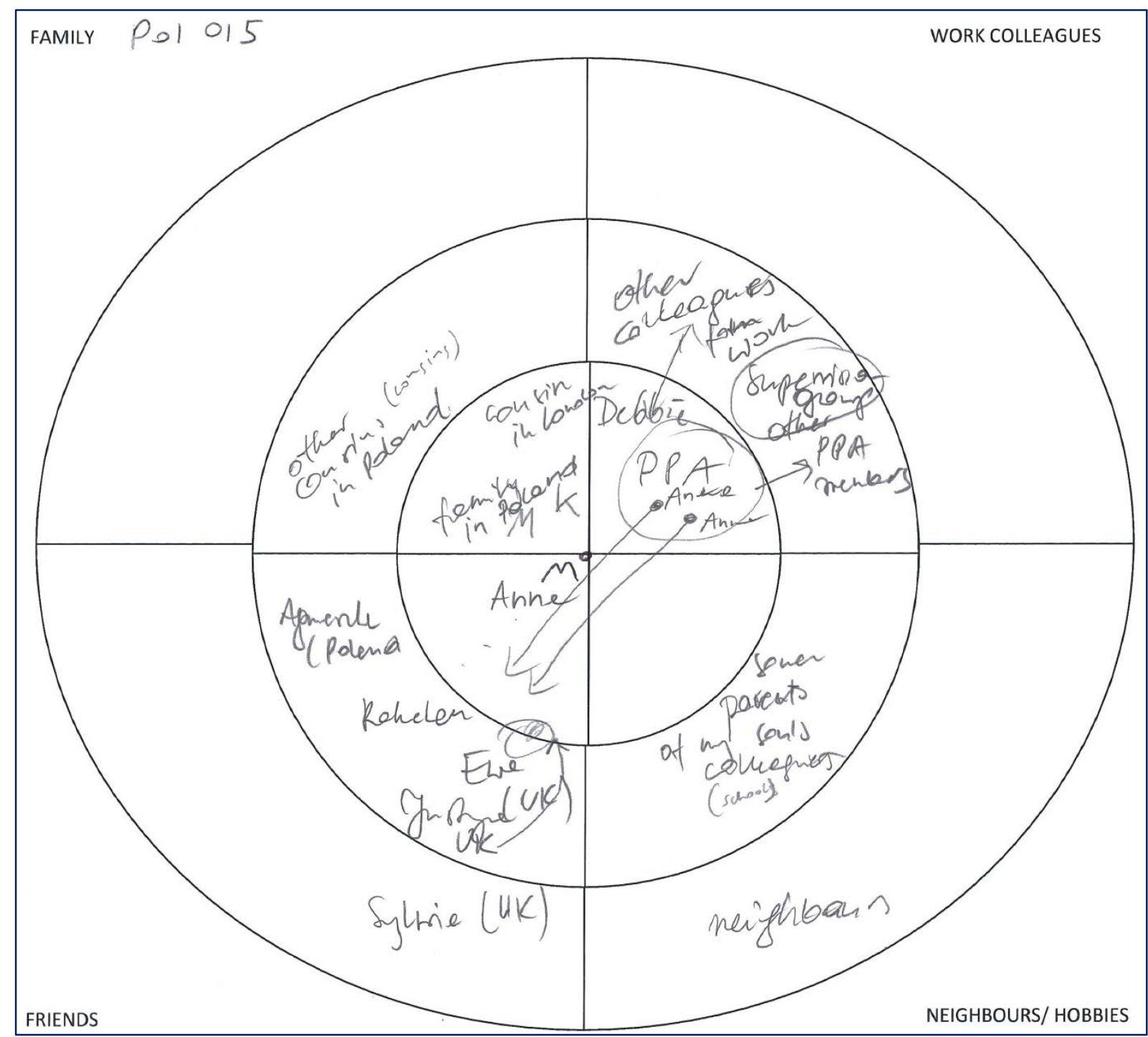

Figure 1: The personal network of Agnieszka, a respondent from the Polish migrants study. Concentric circles represent relational proximity to ego, while quadrants represent context of relationships. 
Watching and listening to the participants as they were confronted with the sociogram, provided insights into how they reacted to and navigated the visualization process. It was also interesting to observe the variable amount of time that participants took to complete the task. Mateusz, for example, was very speedy and finished quickly. Others took much longer and seemed to really consider at great length where to place people. A few participants took a long time because they seemed to have difficulty understanding what was required. Some found the design confusing, while others found the quadrants restrictive and wanted to depict their contacts in more fluid ways. Wiktoria decided to draw arrows to show overlaps across quadrants. Gabi, an accountant, who was familiar with charts and visual displays of data, nonetheless got the wrong impression and began to fill out the hobbies quadrant with activities "yoga" "swimming" rather than the people and relationships associated with these activities. Having given initial instructions about how to complete the sociogram, the researcher watched and waited to see how participants managed the visualization process, but when confusion was apparent she intervened to offer further clarification.

Recall is a key problem for network research (Merluzzi \& Burt 2013). Confronted by the sociogram, many participants struggled to remember all their friends. Several said they should check their phones or their Facebook pages (Marika and Agnieszka). In talking her way through the sociogram, Agnieszka (figure 1) provided a useful insight into how memories may be triggered by association. Describing a friend whose "husband is Asian", Agnieszka was suddenly reminded of another friend who is Asian. Agnieszka was surprised that she had initially forgotten this friend who provided valuable support with childcare and babysitting. This incident suggests that on the spur of the moment when confronted by the sociogram, even contacts with high levels of frequency and important support roles may be temporarily forgotten. While this may be a general observation about memory applicable to all forms of data collection, it is something that researchers should be mindful of particularly when asking participants, within a limited time period, to list their social ties.

Undertaking this visualisation during the interview, rather than ex-post in a computer laboratory (see Hogan et al. 2007) meant that interviewers could hear participants talk through where to place people and how they compared the relative closeness of different people in their circles. In considering where to place some acquaintances, Dominik observed that "they're not my friends, well, I know I spend a lot of time with them, but I do not consider them as friends". Ewa wondered if she was being fair to her friends in Poland: "Actually, I am not fair, I should put them in here (moving them to a closer circle)".

Ryan found the process of moving alters from one circle to another or from one quadrant to another quite fascinating. With pencils, it was easy for participants to change their minds and re-assign people. Usually participants gave a verbal explanation for why they wanted to change the visual image: Karina explained that she was "relegating" one person because she was less reliable than some other friends.

The process of completing the sociogram was interspersed with prompts and questions from the interviewer. Participants were encouraged to reflect upon the patterns emerged in the visual data. As a result, most participants offered an explanation about why their network looked as it did. Izabela explained why she had so many male friends. As an only child who grew up with lots of male cousins, she felt comfortable with male friends. Visualizations, especially when drawn directly by the respondent and combined with interviews, not only offer the potential to add valuable detail on network size and structure but may also serve to prompt stories about particular relationships thus providing a "strong stimulus for the production of narratives" (Hollstein 2011: 15). Upon completing her sociogram Karina paused and observed: "my theory is maybe (I) have capacity for close friends". She went on to explain that one cannot be friends with endless numbers of people but needs to prioritise a particular number and beyond that there is insufficient "capacity" for other people. The account of a network provided by individuals is their perception, a "cognitive map" which is shaped 
by their memories, impressions and opinions (Conway 2014). All this is visible and audible - the process, the time taken, the hesitancy, moving people around, remembering, forgetting, explaining. This would not be apparent if the sociogram was completed alone, online or if the researcher completed it algorithmically based on the interview data.

The network depicted by the participants is a construction emerging from the sociogram and the interview dynamic. In this sense, the visual and oral data appear as entirely interconnected and interdependent, each shaping and being shaped by the other. This has implications for how the data are analysed. Because of an interest in how social ties are depicted through the interplay of the interview questions and the process of visualization, Ryan conducted an integrated analysis in two phases. In the first phase she conducted a narrative analysis of a complete interview transcript and sociogram, focusing on how a participant tells their story in words and images.

This approach was particularly useful for capturing the dynamics of relationships through the lifecourse. One of the criticisms of sociograms is that they offer a snap shot of network composition at a moment in time and therefore are not useful for capturing dynamism over time (Conway 2014). As discussed elsewhere (D'Angelo \& Ryan forthcoming), combining a sociogram with an interview enables that dynamism to unfold through the narrative. Despite criticisms that the visual tool simply offers a static view of social ties fixed in time, Ryan found that the sociogram may act as a prompt to explore how some relations have changed. In completing her sociogram, Oliwia depicted very few Poland-based friends. When invited to comment on this by the interviewer, Oliwia explained how her friends in Poland had weakened over time. She described how these long-distance friendships: "kind of dissolved. When I was going back first, it was always easy then, but you know, my life changed so dramatically, so did theirs, they've got families and children...we didn't understand each other anymore". In this way the narratives and the sociograms combined together provide insights not only into how networks are composed now but also how they have changed over time.

The second phase of the analysis was conducted in NVIVO and involved thematic coding across the full data set to identify the extent to which specific nodes were shared by all participants. This enabled an analysis of particular patterns of networking, such as what factors facilitated new friendship-making in contexts of migration. An interesting theme to emerge across the data was the extent to which becoming parents afforded opportunities to expand one's networks through childbased sociality. For example, the integrated visual and interview data provided by Martyna, Klaudia, Wiktoria and Ewa demonstrate the role of schools and nurseries in providing access to new social networks for migrant mothers.

Thus, this experience suggests that just as visual and narrative data are collected together, there is a strong rationale for analysing them together through this two phase integrated method. This analysis captures the dynamic interplay between how people talk about and visualize their social ties.

\section{Combining participant-drawn and algorithmic visualization}

If the paper-and-pencil target sociogram can usefully accompany the interview encounter, Paola Tubaro's work shows that its computer-based version can enhance the questionnaire experience, increasing response rates and improving data quality without an interviewer. The risk of drop-out is high in self-administered surveys, and would escalate with questions requiring respondents to list members of their networks with all their ties to one another, notoriously time-consuming (McCarthy \& Killworth 2007). Replacing such questions with a sociogram-drawing exercise adds sensory appeal to the response experience and reduces burden, to the advantage of both participants and researchers.

Tubaro and co-authors used such a tool with the difficult-to-reach population that gravitates around so-called "pro-anorexia" (or "pro-ana") websites, set up and maintained by and for persons with eating disorders. Public controversies and threats of censorship surround these websites, accused of 
portraying eating disorders as a lifestyle choice rather than a disease (Casilli et al. 2013). This stigma and health concerns hinder contact with strangers, which is why a self-administered, online survey appeared as a way to minimize intrusion, and a sociogram-drawing tool was embedded in it to limit drop-out. This approach enabled Tubaro's team to hit their target of about 300 completed questionnaires.

The sociogram-drawing exercise required participants to place alters in a concentric-circles structure without sectors (Figure 2), distinguishing different degrees of relational proximity to themselves (intimate, very close, close, not-so-close). They entered name, gender, and type of relationship (such as friend, family member, etc.) of each alter. They could draw ties between alters, group them if they shared some social context of interaction (such as school, family, workplace), and move them around. There were two such sociograms, one for general (primarily face-to-face) relationships and the other for online ones - particularly important here. At the end, participants were asked whom, from among all their alters (shown to them as a list), they would turn to for health-related support.

The software retrieved the underlying data as an edge list -a standard column format for relational data, whereby two adjacent columns contain the endpoints of a list of implied ties, completed by their attributes. Edge lists can be used as inputs in standard network analysis software, to compute metrics such as size (number of network members) and density (number of existing ties relative to all possible ties).

Edge lists capture only objective, measurable information on structure and composition of the network, while drawings also reflect personal perceptions. Participants displayed creativity in their use of the concentric-circles structure to place alters, ties and groups around themselves, and few even used the tool in unexpected and somewhat provocative ways (Figure 2, right panel) - a usage which in itself discloses extra information, but which researchers wouldn't have been aware of, had they stored the data in edge list format only. Conversely, however, edge lists are flexible enough to be adapted to store and reveal key information retrieved from the survey but not displayed in the drawings: in particular, the presence of any alters in both the general and online sociograms of the same ego, the distinction between the supportive alters (named at the end of the questionnaire) and other alters, and the order in which ego entered alters.

We saw above that Ryan made the most of her participants' drawings by analysing them in conjunction with different data - narratives. Similarly, it was necessary here to combine participants' sociograms with other data - edge lists. Tubaro's team chose to do so by sticking to the visual language - transforming the edge lists into algorithm-generated visualizations. Her experience demonstrates that joint use of these two types of visualisation explores and exploits the full richness of the data, supporting not only the data collection, but also the analysis stage of the research. 


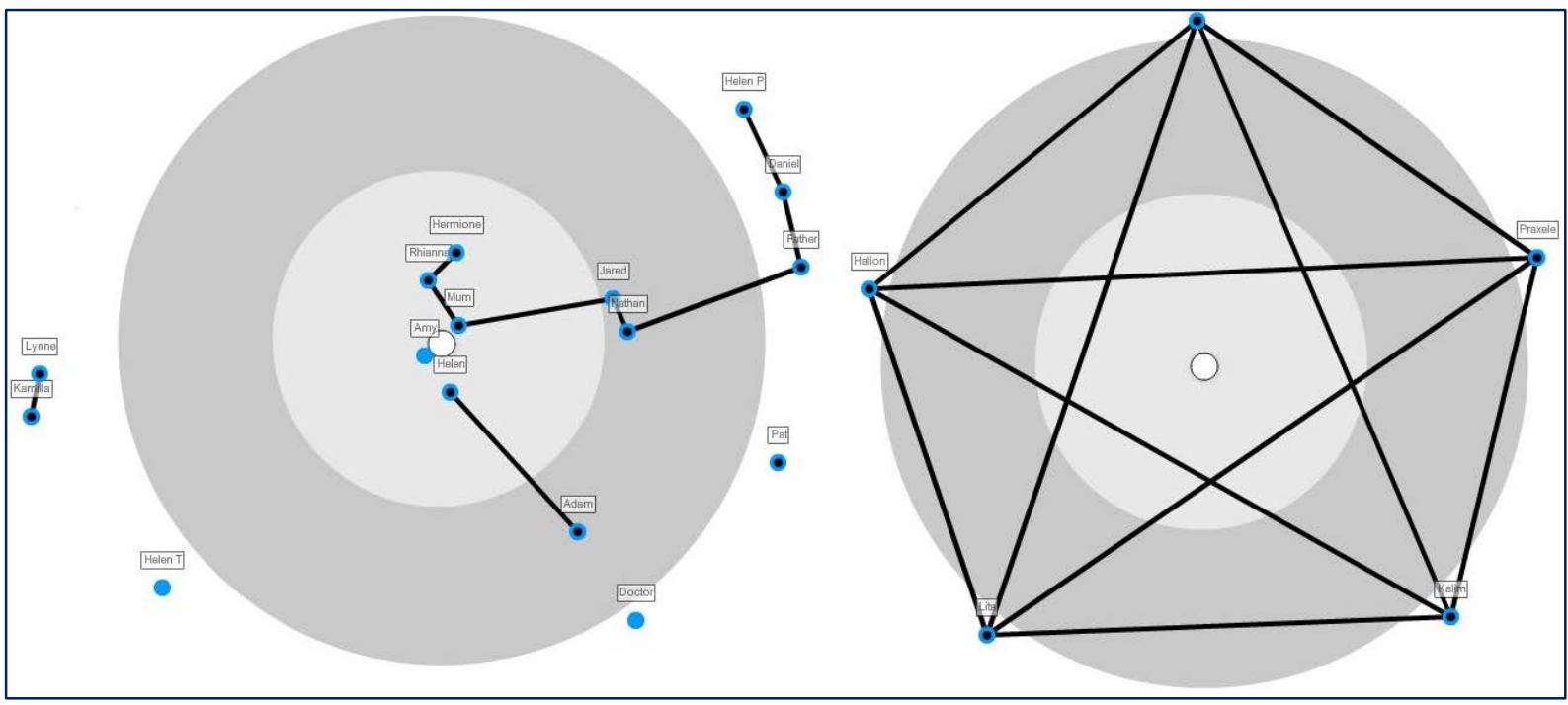

Figure 2: two participant-drawn sociograms, both representing face-to-face networks, from the study of eating disorders. Ego is at the centre (white point), blue points around it represent alters, lines are ties between alters; distance of an alter from ego represents relational proximity as seen by ego. Notice the use of space and lines on the right: a somewhat unorthodox use of the tool.

Figure 3 illustrates a personal network, first in its representation by an individual, who drew a general and an online sociogram (top panels), and then through the algorithm that combined data from the two sociograms (bottom left), as well as the nominations of supporting alters at the end of the questionnaire (bottom right), in a single visual realization. The layout of the algorithmic visualisation still has ego at the centre and alters around, but concentric circles are removed: here, relational proximity is no longer represented as spatial distance from ego, but with a colour coding. The visualisation is dynamic ${ }^{1}$ : alters appear one by one in the order in which ego named them, starting from members of the face-to-face network, represented as hollow rings, to members of the online one, represented as smaller full circles; the two are nested into each other if an alter is present both online and face-to-face. The attributes of an alter can be read by mouse-over. Once the graph is created, a button enables the viewer to identify the supportive alters (the names that respondents had ticked at the end of the questionnaire), who are then drawn closer to ego while all others are moved further away. This algorithmic visualisation loses the immediacy of participants' perceptions and the intuitiveness of the target-shaped spatial structure, but gains in richness of information displayed, in particular as concerns the overlap between online and face-to-face networks. For example, it reveals that this particular participant has several alters with whom contact is both online and face-to-face (such as the two three-cliques in the bottom left panel) and that all supportive alters are drawn from this group (bottom right).

\footnotetext{
${ }^{1}$ For a dynamic video representation of one such personal network, please see https://www.youtube.com/watch?v=EeR0SnFI2M\&index=3\&list=PL61sfC BBbEtOnowIXOpUG0ex3ISgXhxY.
} 


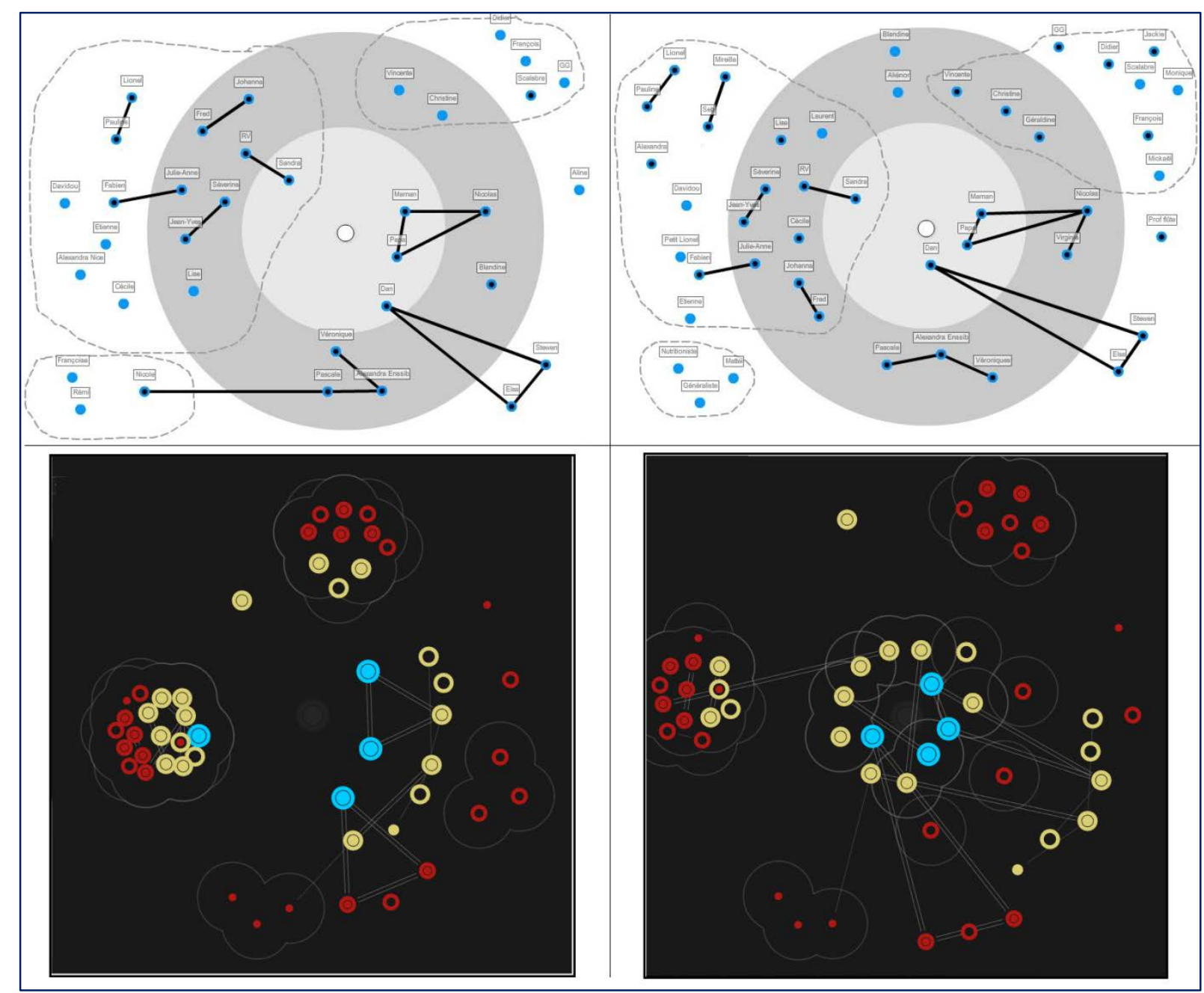

Figure 3: Top: drawings of one participant to the study on eating disorders, representing their face-to-face (left) and online (right) personal networks. Bottom: algorithmic visualization of the participant's face-to-face and online personal networks, combined in a standard layout (left), and with a re-arranged layout that draws supportive alters nearer to ego (right). In all cases, ego is at the centre, small points around it represent alters, straight lines are ties between alters, and curved lines represent groupings. In the top panels, distance of an alter from the centre represents relational proximity to ego. In the bottom panels, colours represent relational proximity (green: intimate, blue: very close, yellow: close, red: somewhat close); the hollow rings are face-to-face alters, the filled points are online ones. The bottom images are snapshots in a dynamic video sequence in which alters appear in the order in which ego originally included them; and are re-organised at the end to highlight those that ego considers as potential support providers.

The ex-post visualisation algorithm applies the same criteria to all personal networks, and therefore produces comparable layouts (Figure 4). It uncovers similarities and differences across personal networks, according to structural and compositional indicators, aside from personal idiosyncrasies. It synthesizes uniformly the rich information contained in the edge lists, while still using the appeal of visualisation that initially motivated use of sociograms in data collection. By changing the visual codes, it pinpoints different aspects of the data, which the participants' drawings might not have immediately revealed. For example, relational proximity is represented as geometric distance (position in one of the concentric circles) in the participant-aided sociogram, and as one of four different colours here: the two convey different sensory impressions, potentially raising different questions and opening different directions for research. 


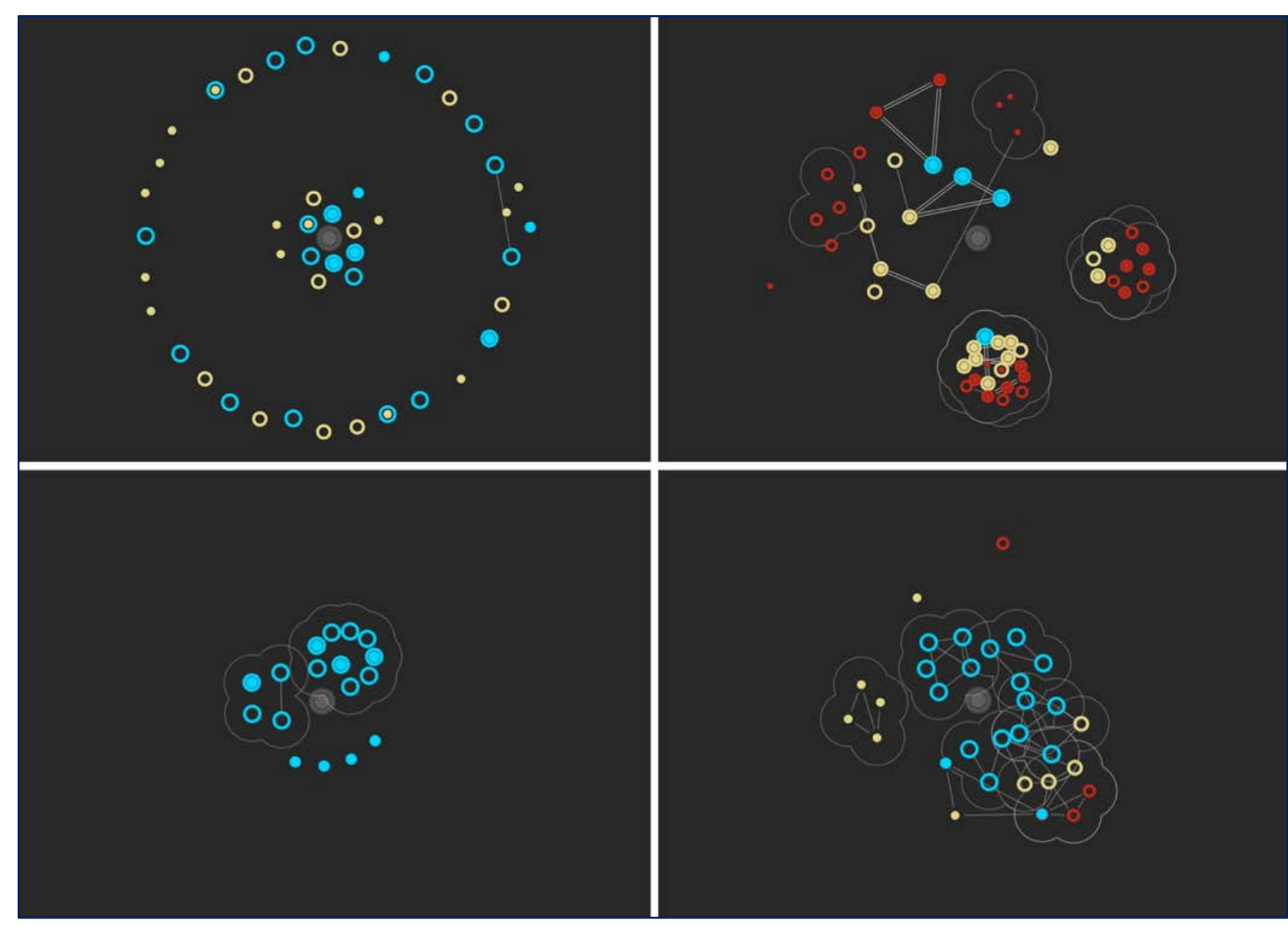

Figure 4: Ex-post visualization of personal networks from the study on eating disorders, from four persons with different eating disorders; coding as above.

The use of participant-drawn sociograms at the data collection stage, and of algorithmic data visualization at the analysis stage, makes understanding more intuitive and aids stronger involvement of external stakeholders - participants and co-researchers. Tubaro's team included colleagues who were fully conversant with relational data and the software to analyse them, and others with no background in this area - who could benefit at most from the visualizations. In this way too, visual tools contribute to opening up research on social networks.

\section{Visualising informal networks through mixed methods}

Our third example pursues our reflection on algorithmic visualization, discussing its applicability not to personal networks but to a "whole network" - the complete set of relationships of a certain type within a specific social context. Whole network sociograms typically exclude unpredictable, idiosyncratic visual inputs from participants and are associated to systematic approaches that construe the research object as a relational structure amenable to automated network visualization. In this sense, whole-network sociograms are sometimes perceived as intrinsically positivistic: ties are either there or not (Crossley 2010). However, in many instances, establishing the presence of ties in a clear-cut and a priori way is not possible - and not even appropriate. How, then, can whole networks be meaningfully investigated in mixed-methods studies, and what is the place of visualization?

The research that Alessio D'Angelo undertook in London between 2010 and 2014 aimed to explore the networking strategies and patterns of local Kurdish community organisations - including advice centres, service providers and cultural associations - and to investigate the extent to which these networks provided individual users with enhanced social capital (D'Angelo 2015). The researcher was confronted with a population characterised by high levels of diversity and internal fragmentation - 
along lines of language, country of origin and political affiliation (Curtis 2005; D'Angelo 2008) - and by a community sector where pressures for professional practices coexisted with high levels of informality (Craig 2011; D'Angelo 2015). This added to the broader challenge of mapping networks of organisations rather than individuals. As Swanson and Holton (2005) point out, organisations are "messy entities": complex, open and dynamic systems. Thus it is important to resist the temptation to anthropomorphosise them (Pennings \& Lee 1999), treating each one simply as a "node", an indivisible and fully coherent unit of analysis.

For all these reasons D'Angelo decided to integrate highly structured and formal techniques, such as matrix- based questionnaires and analysis of official records, with interpretivist methods such as semi-structured and unstructured interviews and participatory observations. This mixed-methods approach produced a wealth of data sometimes in apparent contradiction with each other.

Within this context, the process of visualising the whole network assumed a very particular role. Sociograms did not aim to get an objective representation of a social reality. Rather, D'Angelo sought to produce descriptive outputs, informed by his understanding of networking processes and structures, and attempting to summarise a set of relations as experienced "from within" - thus an intrinsically interpretivist exercise. The development of these charts encompassed an iterative process, where the structural patterns presented in preliminary sociograms informed questions about their content and meaning, and with the results of qualitative methods being used to interpret, but also to enhance and amend the visualisations. After each phase of data collection and preliminary analysis, D'Angelo drew a chart (with the aid of the free social network analytical software Pajek, Mrvar \& Batageli 2015 [1996]) summarising the main ties between organisations as informed by that particular set of data. By looking at different sociograms side by side, he aimed to identify similarities and contradictions and make sense of them.

For example, the analysis of official Charity Commission records to map interlocking directorates (i.e. the linkages that come into being when one person serves simultaneously on the governing board of two or more organisations) indicated an almost total absence of such formal connections, which are widely used in organisational network studies and migrant organisations research (Fennema \& Tillie 2001). D'Angelo's participatory observations within Kurdish organisations revealed how these were superseded by much more informal ties between individual actors operating across organisations often on the basis of longstanding friendships or other personal connections -favouring circulation of information and fostering collaborations of various kinds.

Likewise, the questionnaire generated several non-reciprocated ties, i.e. respondents would report collaboration with some other organisation which, in their answers, did not confirm such links. Interviews with community officers showed how such discrepancies often reflected well-rehearsed narratives, typical of a community sector where demonstration of specific organisational networks is crucial to secure funding ( $D^{\prime}$ Angelo 2015). Moreover, smaller organisations were keener to present themselves as connected to bigger ones than vice-versa. Clearly, the act of completing the structured questionnaire was never neutral, but was part of a presentation of the "networked-self" on the part of individual participants. This made the identification of multiple respondents from each organisation a crucial element of the data collection. Coordinators, members of the board of directors, volunteers and various "community leaders" played different roles and brought very different perspectives on the presence and nature of organisational ties.

There were also instances where the ties which emerged from "formal" data-sets - such as partnerships for externally funded contracts - were not reported by key interviewees, highlighting lack of communication or complex organisational dynamics characterised by conflicting agendas, as exemplified by the coordinator of a Kurdish organisation in North London: "Even though I am here talking with you, what I say it's one thing and what the management committee thinks is something else [laughs]". 
Figure 5, below, presents an example of the sociograms thus produced. It plots the main Kurdish community organisations in London and their reciprocal ties as identified in an advanced stage of the fieldwork. These ties are an overall summary of the strongest and most stable connections between organisations, as existing between 2012 and 2013. A lack of ties does not mean that two organisations have no contacts with each other, but these may be less regular or not related to significant levels of cooperation, exchange of resources or other interaction.

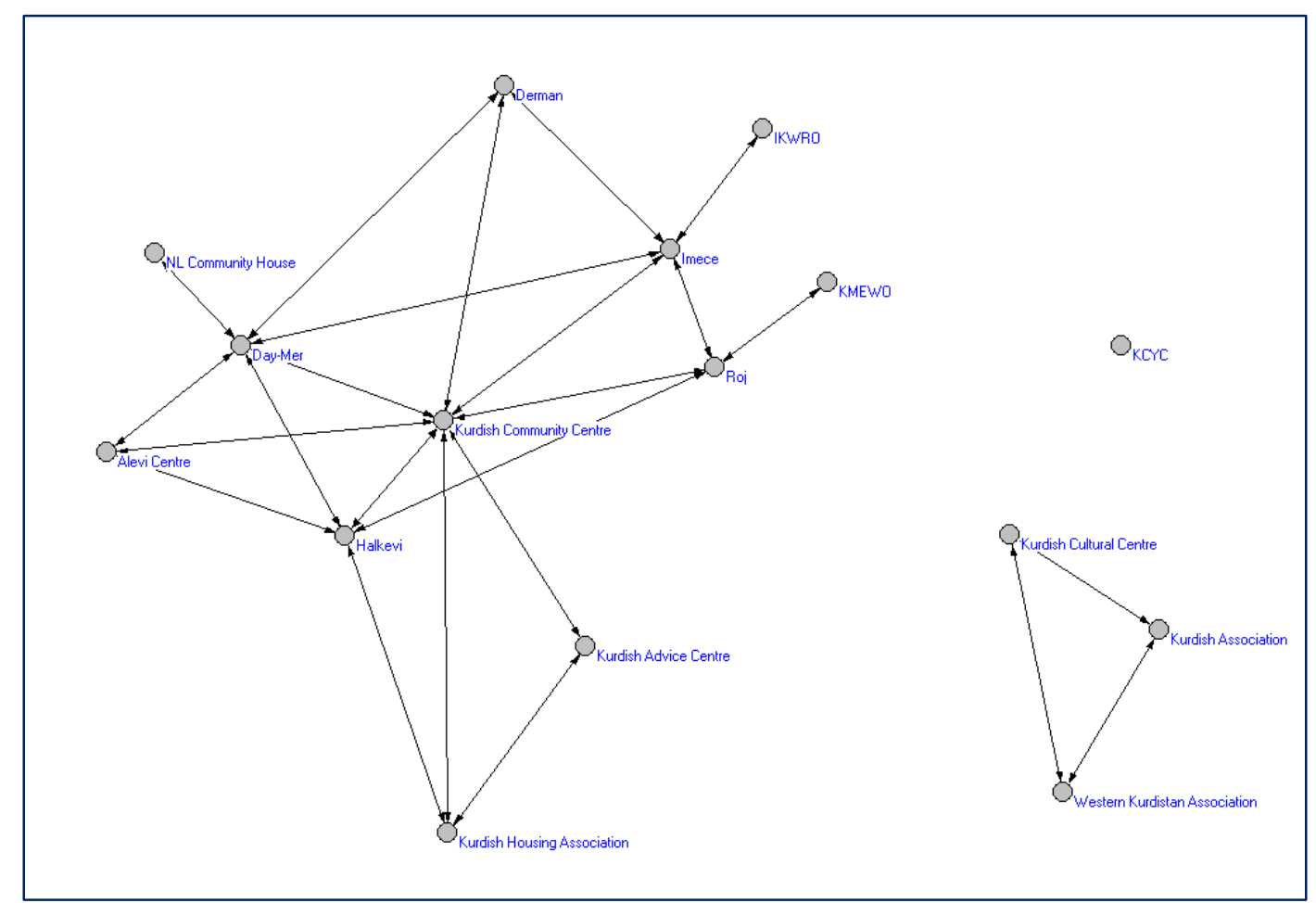

Figure 5: Social network of Kurdish organisations in London (circa 2012-2013).

D'Angelo (2014) provides an in-depth analysis of the networking structures between Kurdish organisations. Here, it is interesting to note the presence of two main "components" (Nooy et al. 2005). The smallest one - on the right in Figure 5 - comprises organisations based in West London and working primarily with Arabic-speaking Kurdish people, mostly from Iraq and Syria, whilst the second, larger component consists of those organisations mainly working with Kurdish communities from Turkey. The presence of "Turkish" and "Arabic" sub-networks is in contrast with the diasporic ideal which some community leaders declare to adhere to and to work for. This division represents the tip of the iceberg of a community which is in fact strongly fragmented in terms of national, political, cultural and linguistic affiliations, and in competition for representation and funding.

Thus, use of sociograms did not just support the interpretation of tensions in the data; it also urged the researcher to constantly think in terms of relative position, centrality and connectivity of individual organisations and to try outlining overall "models" of the network. By visualising his growing knowledge of Kurdish organisations through graphs, D'Angelo would identify - and examine - the presence of specific clusters and sub-groups. Subsequently, these would be displayed in more analytical versions of the sociograms, like the one in Figure 6 , which highlights linguistics groups and political affiliations. Additional visualisations, developed with Geographic Information Systems (GIS) tools, helped explore particular geographical patterns, as in Figure 7 - another example of a substance-based design, matching relational and spatial information. 


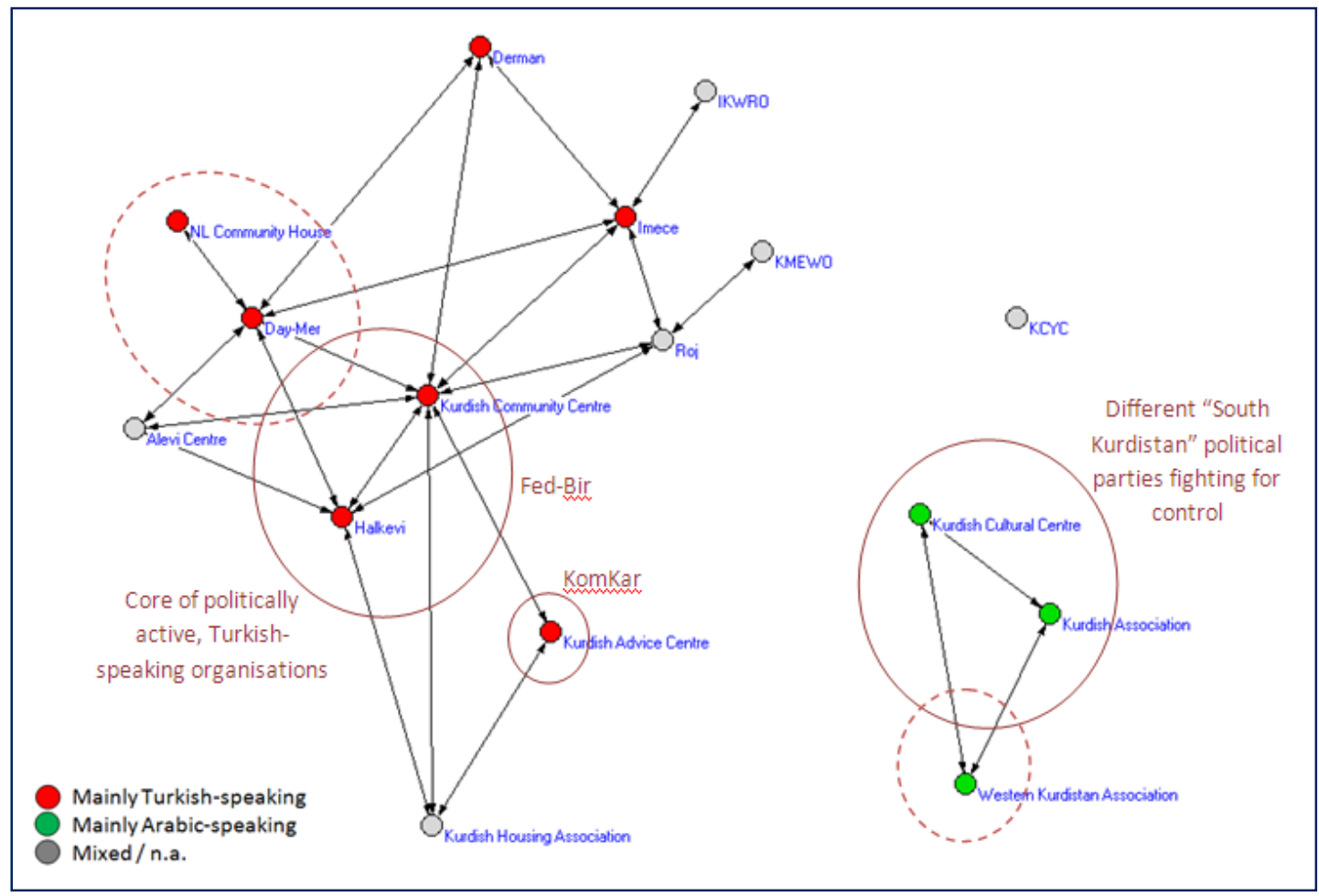

Figure 6: Sub-networks: linguistic groups and political affiliations.

Unlike the case studies presented above, here respondents were not asked to draw their overall view of the network (partly to avoid a mere "representation" of it). However, at a later stage of the study, selected participants saw some of the sociograms and were encouraged to comment on them. The visual image of the network prompted self-reflection. In many instances, the first reaction was to look for the location of one's organisation, to then check which other groups were linked to it. It was rare for respondents to reject the sociograms as inaccurate or "wrong"; however in many cases there was an urgency to "justify" the network's structure, for example why certain nodes appeared to be disconnected from most others.

Some respondents hinted that a lack of inter-ethnic ties can sometimes be counterbalanced by (or be due to) a strong set of links outside the Kurdish community. Specifically, some officers said that it is sometimes easier to establish working partnerships with different Black Minority Ethnic (BME) organisations than with other Kurdish ones, where the above mentioned political and ideological contrasts come into play. As the director of an organisation explained: "We put a lot of emphasis on the importance of partnership with other organisations [...] But in the end we manage to do more with mainstream organisations than with Kurdish ones". In other cases, organisations were more likely to collaborate with those operating in the same area of service delivery - such as housing or legal advice - irrespective of users' ethnic background. Although most respondents recognised a "community of Kurdish organisations" as a social reality in its own right, these results highlighted the limitations of setting network boundaries on the basis of externally ascribed common traits, such as ethnicity (Crow 2004; Alexander 2009).

Other comments referred to the transient nature of ties (Gilchrist 2004), distinguishing between long-term, "strategic" connections on the one hand and short-term, shifting and somewhat "tactical" connections on the other. Even when looking at a sociogram as a snapshot, many organisational links (or lack of them) can only be understood in relation to the history of each group and each individual operating within it. With regard to this important point - which would require further discussion (see Armitage's article in this issue) - the aim of sociograms developed through this mixed-methods approach was not to generate an exhaustive and "final" map of all types of ties existing between 
individual organisations, but rather to provide an overview of selected, specific connections, addressing particular research questions and informing further reflection and investigation.

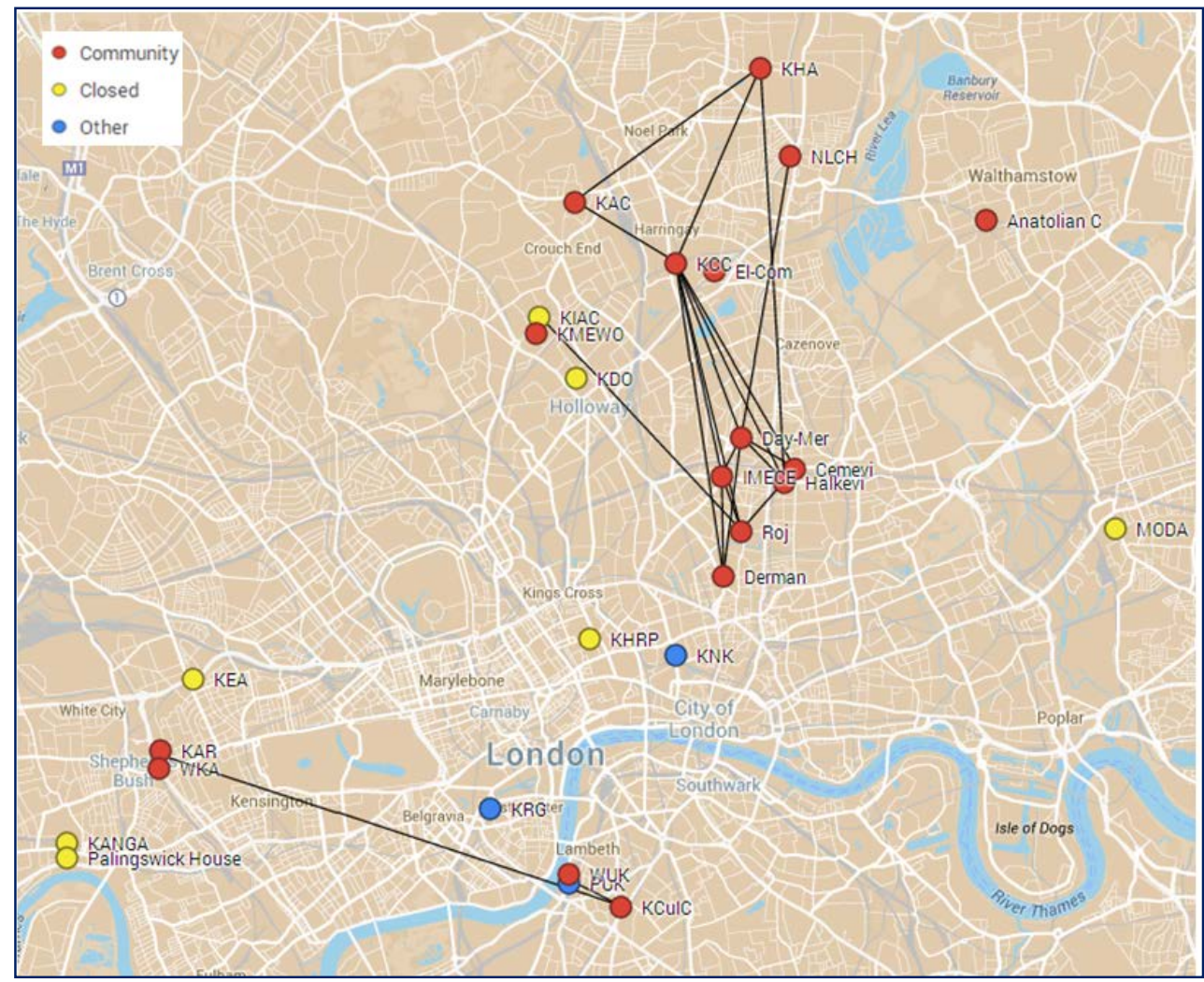

Figure 7: Social network of main Kurdish organisations in London - geographical plotting.

\section{Discussion}

The three case studies presented above exemplify how visualizations can be combined with a variety of different methods, quantitative or qualitative, to gather network data, to analyse them, and to present results. The first two were experiences of using participant-generated, target-shaped sociograms in data collection. Louise Ryan's experience of using sociograms in in-depth interviews, and analysing narratives and images jointly, illuminates the contents, meanings and dynamics of people's relational experiences. The relevance and usefulness of jointly collecting and analysing relational data comes to the fore.

Paola Tubaro embedded similar target-shaped sociograms in a web questionnaire to communicate and engage with a hard-to-reach population, confirming their usefulness as data collection tools. At the analysis stage, the data derived from participants' drawings and their responses to the questionnaire were converted into standardized images through a visualization algorithm. This second sociogram, still centred on the focal individual but eliminating the concentric-circles structure, revealed different aspects of personal networks and aided discovery of common patterns across individuals. Participant-drawn and algorithmic visualizations serve different stages of the research process and enable involvement of different stakeholders - respectively respondents and coresearchers.

Alessio D'Angelo also used algorithmic visualization - not to study personal networks but to reconstitute a whole network. Yet his exercise was far from the standard practice of inputting structured data into a software tool that would map them into images. Because of the informal nature of the empirical context under study, D'Angelo could only collect unsystematic information 
from diverse and somewhat incongruent sources. He thus tweaked the sociogram tool to capture and support his interpretivist exercise, based on his understanding and experience of actors' networking processes. The visualizations helped D'Angelo to progressively develop an allencompassing, coherent view, and feedback from participants further enriched his analysis. Visualizations helped him to mix methods consistently, and to gain insight into otherwise difficult-toreach social contexts.

In all three cases, visualization contributed to different stages of research, including data collection and analysis. Sociograms can be integrated in multiple ways with other analytical approaches, whether quantitative or qualitative, facilitating their dialogue and channelling substantive ideas as well as network insight in harmonious ways.

Mixed methods emerge as the best possible framework to enact the potential of visualization as an addition to the toolkit of the social scientist. In the three cases under study, visualization was integrated with other methods, illuminated the data and analyses, and was in turn informed by them. This substantiates our claim that visualisation has a decisive role to play in mixed-methods social networks studies, over and above its contribution, already acknowledged, to quantitative research.

Visualizations also offer an opportunity to extend social-networks thinking to multiple stakeholders. Sociograms enable respondents to gain a more systematic view of their social environment (Ryan), and to reflect and comment on it ( $\mathrm{D}^{\prime}$ Angelo); they help to approach hidden populations and informal social groupings that standard methods would fail to reach (Tubaro and D'Angelo). Tubaro also argued that visualization helps involving researchers from outside the field, an approach that is still in its infancy, but may help to discover new patterns in the data.

Limitations remain, and this article has no pretension to review exhaustively what can and cannot be done with visual representations of social networks. Particularly relevant are ethical issues. Some are common to all social networks research, notably the impossibility of full anonymity at the data collection stage (Borgatti \& Molina 2003), and the lack of informed consent of (at least some of) the alters nominated by participating egos. Visualization may make these issues more prominent, insofar as graphs disclose alters' names: The researcher must take extra care to further anonymize images before dissemination. Another ethical aspect is the emotional impact that sociograms (whether participant-drawn or realized in the lab from relational data) can have by making participants or other stakeholders feel exposed, or in need of solace: for example, some may experience discomfort at realizing that they lack relationships of a certain category (Ryan et al. 2014).

Researchers should also think carefully about the technical and substantive choices underlying a graphical network representation, and their readability especially for non-specialist audiences. What content is to be displayed, and how? How many concentric circles and sectors should a target sociogram include? Are dynamic formats to be preferred over static ones? What labels have to be shown? If viewers (especially laypersons) are not made aware of at least the basic principles underpinning these choices, they will have limited capacity to appraise visualizations critically. Transparency becomes essential, not only as a pre-condition for scientific rigour and replicability, but also to increase the participatory potential of data visualizations. More generally, the public's education about data - particularly, how to interpret data visualizations - is likely to become a most pressing recommendation if use of visualization in social networks (and other) research has to bloom. 


\section{References}

ALEXANDER M. (2009). Qualitative social network research for relational sociology. In The Australian Sociological Association 2009 Annual Conference. Canberra: TASA. URL: <http://www.tasa.org.au/conferences/conferencepapers09/papers/Alexander,\%20Malcolm\%20pape r\%201.pdf>

ANTONUCCI T. (1986). Measuring social support networks: Hierarchical mapping technique. Generations, 10: 10-12.

BARNES J. (1954). Class and committees in a Norwegian island parish. Human Relations (7): 39-58.

BELLOTTI E. (2014). Qualitative Networks: Mixed Methods in Sociological Research. Routledge.

BELLOTTI E. (2008). What are friends for? Elective communities of single people. Social Networks 30(4): 318-329.

BRANDES U., Freeman L.C. \& Wagner D. (2013). Social networks. In R. Tamassia (Ed.), Handbook of Graph Drawing and Visualization, CRC Press, pp. 803-837.

BRANDES U., Kenis P. \& Raab J. (2006). Explanation through network visualization. Methodology, 2(1): 16-23.

BRANDES U., Raab J. \& Wagner D. (2001). Exploratory network visualization: Simultaneous display of actor status and connections. Journal of Social Structure, 2 (4), URL: <https://www.cmu.edu/joss/content/articles/volume2/BrandesRaabWagner.html>

BORGATTI S.P. \& Molina J.L. (2003). Ethical and strategic issues in organizational network analysis. Journal of Applied Behavioral Science. 39(3): 337-350.

BOTT E. (1957). Family and Social Network. London: Tavistock Publications.

CARRASCO J.A, Hogan B., Wellman B. \& Miller E.J. (2006). Collecting social network data to study social activity-travel behaviour. Paper presented to the Transportation Research Board, January.

CASILLI A.A., Pailler F. \& Tubaro P. (2013). Online networks of eating-disorder websites: why censoring pro-ana might be a bad idea. Perspectives in Public Health, 133(2): 94-95.

CHEONG L., Armour C. \& Bosnic-Anticevich S. (2013). Primary health care teams and the patient perspective: a social network analysis. Research in social and administrative pharmacy, 9(6): 741-757.

CONWAY S. (2014). A cautionary note on data inputs and visual outputs in social network analysis. British Journal of Management, 25(1): 102-117.

CORREA C.D. \& Ma K.L. (2011). Visualizing social networks. In C. Aggarwal (ed.), Social Network Data Analytics, New York: Springer, pp. 307-326.

CRAIG G. (2011). 'Forward to the past? Does the BME third sector have a future? Voluntary Sector Review, 2(3), pp.367-389.

CROSSLEY N. (2010). The social world of the network, Sociologica, 1, Doi: 10.2383/32049.

CROW G. (2004). Social networks and social exclusion: an overview of the debate. In C. Phillipson, G. Allan \& D. Morgan (eds.). Social Networks and Social Exclusion: Sociological and Policy Issues. London: Ashgate, pp. 7-19. URL: <https://www.ashgate.com/shopping/title.asp?key1=\&key2=\&orig=results\&isbn=0\%207546\%20342 9\%209>.

CURTIS A. (2005). Nationalism in the Diaspora: a study of the Kurdish Movement. Utrecht: Universiteit Utrecht. URL: <http://www.wekurd.com/AndyCurtisNationalismintheDiaspora.pdf>.

D’ANGELO A. (2008). Kurdish Community Organisations in London: A Social Network Analysis. London: Middlesex University. URL: <http://eprints.mdx.ac.uk/5539/>. 
D’ANGELO (2015), Migrant organisations: Embedded community capital? In L. Ryan, U. Erel \& A. D'Angelo (Eds.), Migrant Capital. Networks, Identities and Strategies, London: Palgrave Macmillan.

D'ANGELO A. \& Ryan L. (forthcoming), Social network analysis: a mixed methods approach. In L. McKie \& L. Ryan (eds.). An End to the Crisis in Empirical Sociology?, Routledge.

DIAMOND S. (2010). Lenticular galaxies: The polyvalent aesthetics of data visualization. Code Drift: Essays in Critical Digital Studies: cds002, URL: <www.ctheory.net/articles.aspx?id=651>

EDWARDS G. (2010). Mixed-Methods Approaches to Social Network Analysis. National Centre for Research Methods. Working paper 015. URL: <http://eprints.ncrm.ac.uk/842/>

EMMEL N. \& Clark A. (2009). The methods used in Connected Lives: Investigating networks, neighbourhoods and communities. ESRC National Centre for Research Methods Working Paper 06/09, URL: < http://eprints.ncrm.ac.uk/800/1/2009 connected lives methods emmel clark.pdf>

FENNEMA M. \& Tillie J. (2001). Civic community, political participation and political trust of ethnic groups. Connections, 24(1): 26-41.

FREEMAN L.C. (2000). Visualizing social networks. Journal of Social Structure, 1(1), URL: < http://www.cmu.edu/joss/content/articles/volume1/Freeman.html>

GILCHRIST A. (2004). The Well-Connected Community: A Networking Approach to Community Development. Illustrated edition. Bristol: Policy Press.

HEALY K. \& Moody J.W. (2014). Data visualisation in sociology. Annual Review of Sociology, 40: 105128.

HEATH S., Fuller A. \& Johnston B. (2009). Chasing shadows: defining network boundaries in qualitative social network analysis. Qualitative Research, 9(5): 645-661.

HERSBERGER J. (2003). A qualitative approach to examining information transfer via social networks among homeless populations. The New Review of Information Behaviour Research, 4: 95-108.

HOGAN B., Carrasco J.A. \& Wellman B. (2007). Visualizing personal networks: working with participant-aided sociograms. Field Methods, 19: 116-144.

HOLLSTEIN B. (2011). Qualitative Approaches. In J. Scott \& P. J. Carrington (eds.) The Sage Handbook of Social Network Analysis, London: Sage, pp. 404-417.

KITCHIN R. (2014). The Data Revolution. Big Data, Open Data, Data Infrastructures and Their Consequences. London: Sage.

LIU A. (2004). Transcendental data: Toward a cultural history and aesthetics of the new encoded discourse. Critical Inquiry, 31: 49-84.

MANOVICH L. (2002). Data visualisation as new abstraction and anti-sublime. Working paper. URL: <http://manovich.net/index.php/projects/data-visualisation-as-new-abstraction-and-anti-sublime>

MANOVICH L. (2010). What is Visualization? Working paper. URL: <http://manovich.net/content/04projects/064-what-is-visualization/61 article 2010.pdf>

MCCARTY C. \& Killworth P. (2007). Impact of methods for reducing respondent burden on personal network structural measures. Social Networks, 29: 300-315.

MCCARTY C., Molina J.L., Aguilar C. \& Rota L. (2007). A comparison of social network mapping and personal network visualization. Field Methods, 19(2): 145-162.

MERLUZZI J. and Burt R.S. (2013). How many names are enough? Identifying network effects with the least set of listed contacts. Social Networks, 2013, 35(3): 331-337.

MITCHELL J.C. (1969) Social Networks in Urban Situations. Manchester: Manchester University Press. 
MORENO J.L. (1953 [first edition 1934]). Who Shall Survive? Beacon, N.Y.: Beacon House Inc.

MRVAR A. \& Batageli, V. (2015 [first version 1996]). Pajek. Programs for Analysis and Visualization of Very Large Networks. URL: <http://mrvar.fdv.uni-lj.si/pajek/>.

NORTHWAY M.L. (1940). A method for depicting social relationships obtained by sociometric testing. Sociometry, 3: 144-150.

PAHL R. \& Spencer L. (2004). Personal communities: Not simply families of 'Fate' or 'Choice'. Current Sociology, 52(2): 199-221.

PEAY E.R. (1980). Connectedness in a general model for valued networks. Social Networks, 2(4): 385410.

PENNINGS J.M. \& Lee K. (1999). Social Capital of Organization: Conceptualization, Level of Analysis and Performance Implications. In R.T.A.J. Leenders \& S.M. Gabbay (Eds.). Corporate Social Capital and Liability. Norwell, MA: Kluwer Academic Publishers.

PIKE W.A., Stasko J., Chang R. \& O'Connell T.A. (2009). The science of interaction. Information Visualization, 8 (4): 263-274.

RYAN L., Mulholland J. \& Agoston A. (2014). Talking ties: Reflecting on network visualisation and qualitative interviewing. Sociological Research Online, $19 \quad$ (2) 16 <http://www.socresonline.org.uk/19/2/16.html>10.5153/sro.3404

RYAN L. (2011). Migrants' social networks and weak ties: accessing resources and constructing relationships post-migration. Sociological Review 59 (4): 707-724.

RYAN L., Sales R., Tilki M. \& Siara B. (2008). Social networks, social support and social capital: The experiences of recent Polish migrants in London. Sociology, 42: 672-690.

SAMUELSSON M., Thernlund G. \& Ringstrom, J. (1996). Using the five field map to describe the social network of children: A methodological study. International Journal of Behavioural Development, 19(2): p. 327-345.

SWANSON R.A. \& Holton E.F. (Eds.). (2005). Research in Organizations. Foundations and Methods of Inquiry. San Francisco: Berrett-Koehler Publishers.

TAPINI, E. (forthcoming) Intra EU-Highly Skilled Migration and Transnational cosmopolitanism in London, $\mathrm{PhD}$ thesis, Middlesex University.

TUBARO P., Casilli A.A. \& Mounier L. (2014). Eliciting personal network data in web surveys through participant-generated sociograms. Field Methods, 26 (2): 107-125.

TUFTE E.R. (1983). The Visual Display of Quantitative Information. Cheshire, CN: Graphics Press. 